\title{
An overview of the NFAIS conference: Blockchain for scholarly publishing
}

\author{
Bonnie Lawlor* \\ Guest Editor, NFAIS Honorary Fellow, 276 Upper Gulph Road, Radnor, PA 19087, USA
}

\begin{abstract}
This paper offers an overview of the highlights of the NFAIS Conference, Blockchain for Scholarly Publishing, that was held in Alexandria, VA from May 15-16, 2018. The goal of the conference was to take a close look at the initiatives that have emerged as a result of the increasing global acceptance of blockchain technology. This technology, chiefly known as the foundation of Bitcoin and originally introduced as a means of securely managing cryptocurrency, has proven to have practical applications beyond finance. The basic technology is that of a distributed ledger and it is being broadly-adopted by multiple industries, including the scholarly publishing community. The capabilities of this new technology are prompting a direct exchange among stakeholders, as blockchain promises a more structured, decentralized, and immutably secure approach that has the potential to significantly impact researcher workflows - from data collection to peer review to access and published work. The technology inspires passion - there are those who believe that it will ultimately transform our lives while others are completely skeptical. The NFAIS conference provided a look at both sides of the coin (no pun intended).
\end{abstract}

Keywords: Scholarly publishing, Blockchain technology, distributed ledger, citation index, research funding, record keeping, research workflow, decentralized metadata, research attribution, digital object architecture, blockchain for publishing, blockchain for science

\section{Introduction}

I first heard of blockchain technology at the 2017 NFAIS Annual Conference when Christopher Wilmer, Assistant Professor and Managing Editor of Ledger, University of Pittsburgh, gave a presentation on the technology (Note: Ledger is a the first peer-reviewed journal for publishing original research on cryptocurrency-related subjects). While he did mention Bitcoin and other cryptocurrencies, Wilmer's talk was basically on how his journal uses blockchain for proof-of-publication. He commented that as a data-storage mechanism, "blockchains are well-suited to be used in scholarly publishing because they are extremely resilient, tamper-proof, practically indestructible databases; there is no single point of failure or cost of operation; and there is an incontrovertible proof-of-publication date, even across countries and institutions whose incentives are not aligned (which is sometimes a point of contention for scientists racing to discover cure/new theorem/etc.) [1]". I became totally fascinated by the technology and the fact that it was being used outside of the financial realm.

Fast-forward a year later and the momentum of blockchain's adoption was apparent. At the 2018 NFAIS Annual Conference it was announced that a pilot project was soon to be initiated for the

*E-mail: chescot@aol.com.

0167-5265/18/\$35.00 @ 2018 - IOS Press and the authors.

This article is published online with Open Access and distributed under the terms of the Creative Commons Attribution Non-Commercial License (CC BY-NC 4.0). 
development of a protocol where information about peer review activities (submitted by publishers) would be stored on a blockchain. This would allow the review process to be independently validated, and data to be fed to relevant vehicles to ensure recognition and validation for reviewers. Participants in phase one of the pilot include Digital Science, ORCID, Katalysis, Springer Nature, the Taylor and Francis Group, and Cambridge University Press. (You can learn more about the pilot by visiting their website: https://www.blockchainpeerreview.org/).

Why is this technology gaining momentum in its adoption? According to an article in a recent issue of MIT Technology Review, it is because the technology itself " ... is all about creating one priceless asset: Trust [2]". The article talks about the history of the double-entry book-keeping method that dates back to the fourteenth century. It was established as a reliable record-keeping tool and became an integral part of the business culture, but it also allowed financial institutions to become powerful middlemen in global finances - something that continues to this day. But the article also talks about the need to trust such intermediaries and the fact that recent fraudulent activities on Wall Street have significantly reduced society's willingness to trust such middlemen.

The fact is that "trust" is needed in all fields that are "controlled" by middlemen, including publishing. Today peer review has come under attack because of its lack of transparency along with the inability to reproduce the majority of published scientific research results (note: a recent study has showed that only about one-third of research results could be duplicated and that more than fifty-percent of researchers today say that we have a crisis on our hands.) [3]. But blockchain technology - basically a list of transactions is believed to hold the promise of trust even for non-financial "transactions".

According to a recent report from the National Institute of Standards and Technology (NIST), "Blockchains are immutable digital ledger systems implemented in a distributed fashion (i.e., without a central repository) and usually without a central authority. At its most basic level, they enable a community of users to record transactions in a ledger public to that community such that no transaction can be changed once published". That same publication concluded that "The use of blockchains is still in its early stages, but it is built on widely understood and sound cryptographic principles. Moving forward, it is likely that blockchains will be another tool that can be used to solve newer sets of problems... Blockchain technologies have the power to disrupt many industries. To avoid missed opportunities and undesirable surprises, organizations should start investigating whether or not a blockchain can help them [4]".

After attending this conference, I agree wholeheartedly with the NIST report and strongly suggest that you read the 2017 Digital Science report on the use of this technology in scientific research along with the references contained therein [5].

\section{Setting the stage}

The opening keynote presentation was given by Christopher Wilmer, Assistant Professor, Department of Chemical and Petroleum Engineering, and Managing Editor of Ledger, University of Pittsburgh. The focus of his talk was the use of blockchains for record keeping and he gave a compelling case, tracing the history of record keeping from $6000 \mathrm{BC}$. He said that if you ask people to list the most important inventions of human civilization starting from the pre-historical period, most people would probably say fire, the wheel, something about bronze or pottery, then perhaps the steam engine, electricity, and then the Internet. He noted that he believes that more than a few people would leave out writing, and probably more than that would leave out proto-writing (the writing of pictures and symbols that are not quite language). He went on to say that proto-writing was extremely transformative, because it was the earliest form of 
record keeping. And it was in fact two separate inventions, though often talked about as one. The first invention was the concept of writing things down, and the second was the discovery of a durable medium on which to store that information. In a cave ten thousand years ago, someone cleverly used a reed to "write" down his/her thoughts on wet clay and these writings can still be read today. The discovery that wet clay could be used, with a reed as a stylus, for record-keeping was as important as the idea that one should keep records in the first place. But clay was heavy and bulky, and ultimately the invention of paper greatly improved record-keeping. This was followed by the printing press, which allowed records to exist as metal templates and then be used to make paper records.

And skipping many little revolutions along the way, he noted that we now have computers and electronic records... which can be stored millions of times more densely and transmitted effortlessly around the world. He then rhetorically asked the following question, "Is this the peak of record keeping?" and went on to point out the shortcomings of digital records versus paper records.

Just as clay maintained certain advantages over paper (for example, it did not burn), paper has certain advantages over the electronic records that are used today. Paper records are not easily corrupted, and importantly, it is easier to tell when they have been tampered with, compared to electronic records. Because it is so easy to copy and paste digital data, the authenticity and credibility of electronic records are a much bigger and more pervasive issue than existed with paper records. Another problem is that electronic records, even in vast quantities, can be deleted in an instant with the push of a button - not so easy with paper!

Wilmer noted that while record keeping has evolved in the world in general, there is a parallel tradition in academia... which for some reason is fixated on technologies that were developed in the 18th century and has never moved on. He went on to say that when Galileo discovered that the planet Jupiter had moons, he rushed to the printing press to communicate his findings. But what would Galileo have done today? Would he have tweeted it, or just posted his findings on a personal website? Yet today, academics (and Wilmer included himself among these stubborn traditionalists) continue to send their results to a "press" even if it is an online one... and have the text laid out in "pages" in a virtual "book or magazine". . even if such a thing is never printed. He said that it is true that Publishers provide a necessary service in science today, especially timestamping. Anyone can claim on a personal website that they discovered something five years ago, even if it was only discovered five days ago. Publishers act as trusted referees when there is a dispute over who was first to make a discovery. But now we have this new thing, called a blockchain, which infuses digital records with certain properties that are associated with paper (or perhaps even with stone) records: they cannot easily be destroyed and records are provably authentic. It may very well be that use of blockchain technology in providing authentic and indisputable records will bring academic scholars out of the 18th Century at last.

Wilmer went on to briefly describe the technology, saying that it is a loose collection of technologies that offers a new data storage platform - one that is robust and reliable with no central point of failure, and one that is tamper-proof in that the data cannot be changed or erased. He added that blockchain technology is an exciting area and interest in it is rapidly growing around the world, because it has the potential to dramatically improve the storage and tracking of such things as health records, social security information, land titles, Intellectual Property/patents, international borders, stocks/bonds/debts, and money... (i.e., Bitcoin). He then gave a history of the technology, noting that it has its roots in finance and the evolution of e-cash. It is most closely associated with Bitcoin as it serves at its technology platform [6]. He said to think of blockchain as a distributed ledger that can store all sorts of information, not just financial, and added that today blockchain technology is a hotbed of innovation and research. 
Wilmer then noted some of the areas in which blockchain technology could be applied, for example: situations where there is no appropriate, neutral, custodian for data that everybody trusts (e.g. patent disputes); where a custodian can be well-meaning, but unreliable, (e.g. a startup company that may not be around in the future); and situations in which there is an incentive for a data custodian to cheat when the stakes are high (e.g. a doctor being sued in a multi-million insurance claim). He then went on to talk about applications in publishing and used his journal, Ledger, as an example. Founded in 2014, it is the first peer-reviewed journal for publishing original research on cryptocurrency-related subjects and they use the technology as the foundation of its workflow [7]. He said that immutable time-stamping is one of the major advantages.

In closing, Wilmer, briefly mentioned some existing and potential uses for blockchain in research; e.g. near real-time uploading of research results to a blockchain; sharing of data and collaborating with researchers around the world without relying on a centrally-controlled database (e.g., climate science data); and decentralization of citation indexing and the possible enabling of fractional citations. He said that the possibilities are exciting and blockchain technology is worth exploring.

Wilmer's slides are available on the NFAIS website.

\section{Blockchain and the researcher's workflow}

Wilmer's keynote was followed by a panel that talked about how blockchain technology is being used to support a researcher's workflow from start through publication. The first presentation in this session was jointly given by Michael Puscar, Founder and President, Oiga Technologies (see: http://www.oiga.com/), and Neville A. Mehra, Principal, Digital Strategy, Nampora, Inc. (see: https://nampora.com/). The focus of their talk was on whether or not blockchain technology will disrupt the scholarly publishing industry and the topics covered were the challenges facing Scholarly Publishing in 2018; how blockchain can help deal with these challenges; and emerging blockchain-based solutions, some of which were released as recently as in the two months prior to Puscar and Mehra's presentation.

They said that the current challenges that publishers face are protecting their intellectual property from the activities of initiatives such as Sci-Hub [8] and ResearchGate [9]; remaining relevant in the face of rapidly-changing technology; remaining aware of emerging trends in order to set agendas for their conferences; resolving problems inherent in the peer review process; and marketing and sales in a world of declining library budgets. They noted that researchers also face challenges such as the fact that they are only recognized for publishing positive results; the publishing of their research is becoming cost prohibitive (e.g. Article Processing Charges for Open Access can be as high as \$6,500); they must race to publish before another researcher pre-empts their discovery; it takes an average of six years for a researcher to get their first citation; and that there is a reproducibility crisis in academia because insufficient information is provided in the material that is published.

They then moved on to blockchain, first stating that it is not a cryptocurrency; it is neither a content repository nor software; and it is not owned by any one organization. Most importantly, they emphasized that blockchain should not be a solution seeking a problem. Blockchain is, they went on to say, a digital ledger that is distributed, immutable, secure, decentralized, and time-stamped. They then asked a question: What if an immutable, time stamped registry (ledger) existed that could record every artifact of a researcher's work, with nodes dispersed among research institutions, universities, publishers, and funders - indeed a shared ledger that had pointers to every piece of research that is produced in the world? Their answer was that such a ledger would allow researchers to protect their Intellectual Property 
and conclusively prove their ownership with a timestamp; selectively share their research output with others in the research community, their funders, and with publishers. In addition, funders could monitor the progress of their researchers; other researchers could reproduce experiments more easily; and nodes could be distributed throughout universities, as well as throughout research, publishing, and funding organizations.

They noted that a blockchain ledger confirms discoveries with an immutable time and date stamp and allows the researcher to control what parts of discovery are public or private and with whom they are shared. Every experiment, lab note, formula, etc. is logged on the blockchain ledger along with all subsequent versions, facilitating more collaboration within the research community. A researcher's output can be logged on the blockchain ledger prior to publication and he/she can give permission for funders to have instantaneous access to research as it evolves, along with access to activity reports that highlight any new discoveries. Discoverability of research in progress offers a window for publishers not only to analyze emerging trends, but also to find researchers and solicit them to submit manuscripts to their journals or to serve in the peer review process. The technology can also work in the journal/article purchase process with each purchase being a smart contract on the ledger, allowing pirated versions outside the platform to be traced to the original buyer. Indeed, the ledger would serve as the glue to every content and media asset.

They then provided current applications of the blockchain ledger that they described above. The first was ARTiFACTS (see: https://artifacts.ai/), a new company that is building the "ledger of record for research". It is focused specifically on researchers and scholarly publishing. All artifacts of research will be linked and searchable. It will allow researchers to prove ownership and existence of their work. And it hopes to improve discoverability throughout the research process, with permissioned nodes at institutions (see a separate article on this new company elsewhere in this issue of Information Services and Use). Their second example was Po-et (see: https://www.po.et/), a company that is building "a shared, open, universal ledger designed to record metadata and ownership information for digital creative assets and aims to create the largest verifiable record of digital media assets". Po-et will allow content creators to issue licenses on the blockchain ledger, and appears to be compatible with all types of content (blog posts, videos, etc.).

Their third and final example that was presented is the initiative to use blockchain technology for the peer review process which I mentioned earlier in this article, the goal of which is to make the peer review process more transparent, recognizable, and trustworthy (as a reminder see: https://www.blockchainpeerreview.org/).

Both Puscar's and Mehra's slides are available on the NFAIS website.

The next speaker was Jason E. Barkerloo, Founder and Chair of Knowbella Tech (see: https://www.knowbella.tech/), an organization that connects scientific researchers with funding sources via blockchain technology. He spoke specifically about using the blockchain and smart contracts to manage grants. "Smart contracts" were defined as a computer program that is stored and runs on the blockchain. The contract defines a set of rules and procedures that relate to a binding agreement between parties just like a traditional contract, but unlike its paper counterpart, it can automatically enforce these obligations. An example of one of the smart contract rules with regards to a grant can be when the grant starts, ends, etc., and he noted that smart contracts can improve grant funding by changing the funding model for Open Science. He said that there are many steps in the grant process, including application submission and review, the selection step, background checks, and ongoing management of the grants that are awarded. He believes that use of blockchain both simplifies and speeds-up the process.

Barkeloo referred to Knowbella's U.S. patent filing number 62/555,989, Systems and Methods for Providing a Decentralized Platform for Connecting Members of an Open-Science Community. He briefly 
described the decentralized blockhain-based platform as one that: (1) focuses on connecting members of an open-science community; (2) connects researchers with collaborators and/or funders; (3) generates decentralized electronic contracts; and (4) tracks the progress of research projects and directs payment of funds in awarded tranches.

Smart contracts are prepared as follows: first, the number of research tranches (steps that will require funding) are defined and each tranche has a defined budget, time-frame, and a description of deliverables; second, immutable copies of the original grant proposal are referenced using either IPFS or SWARM both hypermedia protocols for a decentralized system; and finally, all grant funds are stored as a HELIX "token" (see: https://www.thehelixtoken.com/) that converts to a stable coin in order to avoid foreign exchange risks. Once executed, the contract is sent to the blockchain and starts immediately or on some pre-determined date. The life cycle of a specific grant "tranche" is as follows; (1) funds are distributed to the researcher for that specific tranche; (2) the research is performed; (3) the results are published; and (4) the grantors vote to accept the results and approve moving on to the next tranche. The researcher then obtains payment (a token) for his/her work. The process then continues until all tranches have been completed or until some failure occurs; e.g. there is an unexpected discovery, the contract has run out of time, or there has been a failure to vote. The safe exit is that remaining funds are returned to the grantors.

Barkeloo said that the benefits of this new funding model are a reduction in overhead costs related to managing grants, an increase in the speed of awarding grants, and an increase in research collaboration. He closed by saying that Knowbella Tech disrupts the paradigm of siloed, cumbersome processes with an Open Science platform powered by distributed blockchain technology. It opens up dormant scientific intellectual property, enables scientific collaboration around the globe, allows researchers to share their research immediately, and gives grant funders and applicants direct access to one another.

Barkeloo's slides are available on the NFAIS website.

The next speaker was Mads Holmen, Co-founder and CEO of Bibblio (see: https://www.bibblio.org/) who spoke on the use of blockchain technology to unlock and power discovery between publishers for shared users. He said that all the power on the internet belongs to the likes of Google, Facebook, Apple, and Amazon (the Big Four), largely because they control content discovery. Combined, they generate $75 \%$ of all web traffic to websites and close to $50 \%$ of content sales, yet content is not their main source of revenue (advertising is). Users return to them time and time again instead of browsing around the web as it was originally intended because the mode of access is direct and trusted and because these developers built clever technology and algorithms that keep their users hooked. All of these organizations use siloed information and data that is essentially provided by publishers who themselves have built websites, platforms, and apps with walls around users. The Big Four all began with a focus on the user and have since moved into distribution, packaging, and now seem to be marching towards content creation. $\mathrm{He}$ noted however, that while the "Big Four" are all currently weak in content creation, Amazon has moved into this area via movies and books.

Holmen showed a photograph that appeared on the cover of National Geographic and that has become so familiar that just about anyone will immediately recognize it. But it is identified with National Geographic and not the photographer. He said that in some cases, as with this photograph, the middleman controls the value of creativity and blockchain has the potential to eliminate them. He does not believe that individual creators of content such as photos are ready (yet) to use blockchain. But he does believe that blockchain and scholarly publishing are a perfect fit.

He then talked about how blockchain technology provides an opportunity for publishers to take back power from the Big Four by decentralizing the internet once again. The technology addresses distribution. It can drive efficiencies across the value chain, it can increase trust, and it can minimize piracy. He said 
that he believes that the time is ripe for publishers to focus on their content creators. Publishers spend a lot of money working with creators (authors, photographers, video producers, etc.), but at the moment the Big Four build their user loyalty on the back of that content. To a certain extent publishers are reliant on Google, Facebook, and Amazon to direct users to their sites and even drive sales. For publishers to drive increased revenue directly to themselves and their content creators is the real challenge. He suggested that perhaps a flourishing ecosystem model in which publishers collaborate to focus on content creators and information consumers is, at least in theory, more economically efficient than the status quo, but in closing noted that to do this will require much more industry collaboration, an ecosystem mentality, and a real focus on content creators.

Holmen's slides are available on the NFAIS website, and a brief article based upon his presentation appears elsewhere in the issue of Information Services and Use.

The final speaker of the day was Tyler Weirick, PhD, Senior Research Technologist, Division of Cardiovascular Medicine, Cardiovascular Innovation Institute, University of Louisville, KY, who spoke on adding blockchain functionality to computational workflow-managers. Specifically, he discussed a new product, MakeElevator, designed for use in bioinformatics, an interdisciplinary field that uses large amounts of data and computer power to study issues in the biological disciplines.

He said that MakeElevator is a blockchain-based protocol for communicating computational workflows. It increases the speed, reproducibility, and accessibility of computational workflows and is intended to be used with "workflow managers". The product's name comes from a combination of Snakemake (a workflow management system [10]) and space elevators (a proposed type of planet-to-planet transportation system [11]). It consists of the following: a set of rules for formatting and organizing input and output data; a set of rules for controlling write access to the blockchain; the InterPlanetary File System (IPFS), a protocol that makes it possible to distribute high volumes of data with high efficiency (see: https://ipfs.io/); and a Bigchain Database. The latter is a scalable blockchain database that is said to merge the best of both the distributed database world and the blockchain world, for while the database scales and is queryable, it is also decentralized (no single entity owns or controls it), immutable (tamper-resistant), and the assets are yours if you own the private key (see: https://www.bigchaindb.com/).

The product functions by associating hashed key-value pairs, and while designed for bioinformatics, Weirick said that it could also be interesting for publishers. MakeElevator uses the IPFS to create hashes for the input and output of GNU-Make-style "rules" and publically stores them in a blockchain. But once in the blockchain, this information can be used for many purposes. Since all rules and data are easily obtainable and data and even whole pipelines can be communicated within articles as hashes, re-analysis can be accomplished more easily and quickly, ultimately improving the overall reproducibility of research results.

Weirick gave a demonstration of the product and then went on to say how it might be useful for journals. He believes that journals add value to reported research as a result of the publication process - peer review, copy editing, clarity of layout, etc. Until recently, journals also added value by warehousing data, but this is has become less important since it is increasingly easier to host large amounts of data online. MakeElevator can improve the quality of computational analyses that are included in journal articles because the exact details and files for each step are easily and instantly accessible (the hashes of entire analyses can be described within a journal article). Basically, it helps increase the quality of bioinformatic analyses, improves reproducibility, and reduces file hosting costs. The product also helps scientists, as it offers faster computational pipelines, a new way of getting attribution for their work (especially for currently untracked, but useful contributions). And it is possibly monetizable in the future.

Weirick's slides are available on the NFAIS website. 


\section{Shared, decentralized library repositories}

The second day of the conference opened with a session on decentralized repositories. The first speaker was Jason Griffey, Affiliate Fellow at Berkman Klein Center for Internet and Society, Harvard University. He opened by saying that with regard to blockchain technology we are at the height of the technology hype cycle - the section of the curve that is known as the peak of inflated expectations [12]. He said that the term "blockchain" is highly-recognized and used a great deal, but it does not communicate much concrete meaning. It is today's technology buzzword. However, he noted that blockchain and other modern decentralized technologies $d o$ have the potential to fundamentally change the flow of the information economy, for blockchain is, at its core, a technology that distributes power... what happens when the varieties of power in information flows are disrupted?

A core attribute of blockchain is the fact that it is a decentralized system and therefore the roles of librarians and publishers are at question, for power will no longer reside in traditional places. The "middleman" as Mads Holman pointed out earlier, will become redundant. He noted that today good provenance is accomplished by the use of metadata that reflects the chain of ownership of files and physical objects. It facilitates the tracking of files over time, and it is pretty much under the control of the publishers. This continues to be the case even in today's digital world. But enter blockchain and there now becomes the potential for users to create and control their sovereign identity along with the capability for distributed verification. He described it as a "universal library card" which will allow information consumers to own their identity and move between information systems. The "card" would be the means of authentication. He said that this would pretty much facilitate access to the world of information and would also make license agreements difficult to create and enforce. He then talked about the potential for a blockchain-based metadata system - sort of decentralized OCLC - that could also be put into the hands of information consumers and users.

He noted that perhaps of more concern to publishers is that blockchain can support the first sale doctrine. Codified at 17 U.S.C. $§ 109$, this doctrine states that an individual who knowingly purchases a copy of a copyrighted work from the copyright holder receives the right to sell, display or otherwise dispose of that particular copy, notwithstanding the interests of the copyright owner. The use of a metadata-based blockchain and the use of bitcoins could facilitate and verify such a sale and would open up a secondary market for all information that is actually sold (as opposed to being licensed). He commented that if metadata is decentralized the cost actually may be net-neutral and the blockchain could become a "one stop" shopping point for information users rather than having to go to multiple publishers (again building on Mads Holman's suggestion that publishers work together to focus on the information user/consumer).

Griffey then discussed a year-long IMLS-funded project that has the goal of gaining a better understanding of blockchain technology and imagining its potential for small and large, urban and rural libraries and their communities (see: https://ischoolblogs.sjsu.edu/blockchains/). The site has a link to videos that not only explains blockchain technology, but also discusses potential applications - definitely worth a look.

In closing, Griffey said that blockchain technology is exciting: it removes centers of control and distributes power; it democratizes decisions; and it reduces friction - it is a major potential disruptor. As a final reality check for meeting attendees he cited Amara's law: "We tend to overestimate the effect of a technology in the short run and underestimate the effect in the long run [13]".

Griffey's slides are available on the NFAIS website.

The next speaker in this session was Lambert Heller, Head of Open Science Lab, TIB - German National Library of Science and Technology, Hannover, Germany, who discussed how advanced Peer-toPeer (P2P) Architectures will set new standards for how we support scholarly works and interactions. He 
said that today we have a problem with research assessment and the challenge to give researchers "agency", which he defined as direct control over their identities, assets, and interactions. Some of the elements of research assessment are peer review, giving attribution, (including attribution for minor contributions to a collaborative work), or other forms of mutual (micro) assessment. He noted that this problem is a systemic one, for most critical committee decisions such as hiring, promotion, tenure, funding etc., still use flawed "proxies" such as the journal impact factor for assessment. He offered the emergence of ResearchGate (see: https://www.researchgate.net/) as an example of how we have failed to give researchers agency and transparency regarding mutual assessment. He said that ResearchGate, and to some degree academia.edu (see: https://www.academia.edu/), have captured the assessment market for now, with both services mimicking Facebook's business model. He noted that the outcome of their assessment is highly ambivalent, and suggested that attendees take a look at the RG Score [14] (a measure of how a scientist is perceived by his/her peers) and at the recent ResearchGate/Springer agreement. The latter refers to the fact that Springer Nature and ResearchGate, along with Cambridge University Press and Thieme, have agreed to work together on the sharing of articles on the scholarly collaboration platform in a way that protects the rights of authors and publishers (see: https://group.springernature.com/gp/group/media/pressreleases/springer-nature-and-researchgate-announce-new-cooperation/15705990).

Heller also cited ORCID as an example of how we have failed to give researchers agency and transparency regarding mutual assessment. ORCID is not a primary place where input comes directly from researchers. It is, for the most part, seen and used as an aggregator that collects metadata and that is controlled by big publishers. He added that while ORCID represents real progress and is indispensable, it has systemic metadata quality issues, and it is currently not a tool that gives researchers and contributors ultimate control over attribution, mutual assessment, etc.

So how to fix the problem? He back-stepped to say that in 2016 the MIT Media Lab, together with Learning Machine, came up with the idea of "blockcerts" (see: https://www.blockcerts.org/). This concept had students establish their own unique identity on a blockchain and when it was time to graduate they could quickly and easily get a verifiable, tamper-proof version of their diploma that could be shared with employers, schools, family, and friends, giving them autonomy over their records. Heller said that a similar approach would work to give researchers and contributors ultimate control over attribution, mutual assessment, etc. Some building blocks include decentralized identifiers (DIDs) and Self-Sovereign Identity. He noted that everyone can join a public peer-to-peer network (a "blockchain"), set up a node for a particular transaction (i.e. pull in a "prove" for some claim). Claims are cheap, but not free, and therefore few economic assumptions and dependencies would come into play; e.g., "piggybacking" on a growing ecosystem e.g. of crypto wallet apps, blockchain browsers, etc. He added that he realizes that blockchain is still experimental and hyped, yet this approach might be the best candidate to solve research metadata ownership.

But, he continued, researchers' agency is about far more than assessment. It enables self-governed research, disrupting the current system. Today's research system is facing a growing amount of bureaucracy and friction in the way it funds and governs research. As noted by Jason Barkeloo earlier, processes are often highly-intransparent, slow, unreliable, excluding, and vulnerable to interferences from outside of researchers' self-governance. Tokenized research allows researchers - and virtually anyone interested in doing (citizen) science - to literally own their research projects, set up and conduct their own governance rules, and ask for highly-specific, frictionless and transparent (micro)funding from funding agencies and from other sources.

In closing, Lambert said that he believes that blockchain offers a route to a true scholarly commons and that use of decentralized networks to share data and publications could make research more open, efficient, 
and fair. He referred attendees to read one of his articles covering some ideas from his presentation along with further links that is available at https://bit.ly/blockchain-commons.

Lambert's slides are available on the NFAIS website.

\section{Building a system for researcher recognition and citation}

The next panel session focused on building systems for researcher recognition and citation. The first speaker was Richard Ford Burley, Deputy Managing Editor, Ledger, who spoke on the features and drawbacks of centralized citation indexes (e.g. the Science Citation Index ${ }^{\circledR}$ ), ultimately asking whether or not we can leverage incentives to create a decentralized citation ledger using blockchain technology? $\mathrm{He}$ quoted Eugene Garfield, creator of the $S C I^{\circledR}$, as saying, "Whether or not citation indexes are useful is a question that has now been answered... However, a citation index must meet the same economic test that all products in our society must meet: Does the cost justify the benefits [15]?" The $S C I^{\circledR}$ is now part of Clarivate Analytics' Web of Science. He noted that Elsevier has a similar product, Scopus, and Google has Google Scholar. These three products are run by big businesses who must be concerned about their bottom line. Therefore, he asserted, economics must come into play when they decide what material does or does not get indexed in these services.

Burley then cited Bradford's Law of Scattering which is as follows: "Articles of interest to a specialist must occur not only in the periodicals specializing in his subject, but also, from time to time, in other periodicals, which grow in number as the relation of their fields to that of his subject lessens, and the number of articles on his subject in each periodical diminishes [16]". He noted that Garfield had his own Law, the Garfield Law of Concentration; e.g. "In opposition to scattering, a basic concentration of journals is the common core or nucleus of all fields [17]'. Burley said that Garfield added in that same article that "Any abstracting or indexing service that ignores Bradford's Law in attempting to realize the myth of complete coverage does so at its great financial peril". So in light of Garfield's own words, economics does come into play in order to support the efforts and infrastructure required to create such products and services.

Burley said he believes that relying on Garfield's Law of Concentration to provide the most coverage for the least cost creates a self-fulfilling prophecy in that it generates a body of publications that draws scholarship away from less- and non-indexed publications, thus making it more likely that the most important papers will show up primarily in journals that are covered by abstracting and indexing services. He said that he understands journal selectivity is perceived as a necessity for ongoing sustainability, but questioned whether it is necessary in light of today's technology - specifically blockchain. He then went on to describe how a decentralized citation index built on a blockchain might work with authors themselves inputting the necessary information - not employees of a large organization. He views the potential benefits are that such a system would eliminate the pressure on start-up and smaller journals; create a more accessible ecosystem for data curation businesses; and perhaps offer the potential for more targeted curation for social ends. However, he did say that there are potential issues, for example, dishonest scholars and journals would require mitigation by curators; spam attacks would require "captcha"-like proof-ofhumanity or other mitigation techniques; and there would be the need to implement the blockchain and a create a new cryptocurrency or use an existing one such as bitcoin to reward participants. Burley closed by saying that there is a lot of work that would need to be done toward creating a stable, decentralized citation ledger, but such a venture offers very real promise for the future. 
Burley's slides are on the NFAIS website and a more detailed article based upon his presentation appears elsewhere in this issue of Information Services and Use.

The second speaker in this session was Courtney Morris, President and Co-founder of ARTiFACTS, a company launched in March of this year that is using blockchain technology to build a ledger for research (see: https://artifacts.ai/). He said that his company is reframing indexing and attribution by returning to the original vision of Eugene Garfield. He then quoted Garfield as saying that the Mertonian (referring to Robert K. Merton) description of normal science describes citations as being the currency of science. Scientists make "payments", in the form of "citations", to their preceptors. This description, in Merton's own words, is clearly-stated in the Forward that Merton wrote for inclusion in Garfield's book on citation indexing [18].

Morris said that today, the digitization of linkages across research artifacts is captured for only a fraction of outputs, focused largely on published articles, in deeply retrospective, inconsistent and error-prone indexes. ARTiFACTS provides researchers with a simple, user-friendly platform for sharing their research, establishing and maintaining all linkages prospectively, from the earliest stages of research. Through its distributed ledger and transaction engine, it records a permanent, valid, and immutable chain of records for all research artifact "transactions", including citing transactions. He added that ARTiFACTS brings a new level of capability with which to authoritatively and rigorously manage citations, artifact linkages, and ownership pre- or post-publication. In many ways his description of the service echoed Burley's earlier vision (at least with regard to citations) of putting control into the hands of the content creators.

ARTiFACTS focuses on three element: (1) research collaboration via a workflow management and collaboration tool leveraging an open source platform that allows researchers to manage projects and teams and share research findings, giving creators control of their content and instantly establishing proof-of-existence; (2) attribution using an ethereum blockchain engine that records a permanent, valid, and immutable ledger of record for all research findings and their cited/citing attributions, building a comprehensive, real-time, profile of scholarly contribution; and (3) indexing, offering a community index of all research artifacts and linkages that speeds research by allowing discovery of the full spectrum of research outputs, not just published works.

Courtney's slides are on the NFAIS website and a more detailed article based upon his presentation appears elsewhere in this issue of Information Services and Use.

The next talk differed from all prior presentations. The speaker was Kent Anderson, CEO, RedLink (https://redlink.com/), who based his talk on an article that he wrote for the Scholarly Kitchen blog [19]. He opened with a quote by Paul Ford that appeared in Business Week, "Most things that the blockchain promises to do can be done more easily with other technologies, including good ol' fiat currency. But I know a mind virus when I see it [20]". The quote set the tone for the comments that followed.

Anderson went on to say that blockchain is not new and has actually been around for a decade. He said that in almost all cases where it is being used there are better technology alternatives. He added that he believes that there are potentially huge societal downsides to a world that behaves like blockchain and that, if pursued, it could undermine important social constructs that depend on identity, trust, secrecy, and verifiability.

Anderson then went on to talk about blockchains and voting. He said that in a typical election setting with secret ballots, we need: 1 . Enforced secrecy: a way for each voter to cast a ballot secretly and no way to prove how they voted (lest they be unduly influenced); 2 . Individual verifiability: a way for each voter to gain confidence that their own vote was correctly recorded and counted; and 3. Global verifiability: a way for everyone to gain confidence that all votes were correctly counted and that only eligible voters cast a ballot. Blockchain does not help tally secret ballots in a publicly-verifiable way. It does not provide 
individual verifiability that a ballot was correctly encoded. And it is not useful for voting eligibility, since that's all about human authentication and a centrally-produced voter list. At best, in voting, blockchain can be a ledger that helps us track the voting metadata [21]".

Anderson went on to say that one of the key "advantages" that is hyped about blockchain is that it offers decentralization. His opinion on decentralization is that is it neither more cost-effective nor more secure. Its performance is limited by network latency, and secondary systems become major concerns requiring separate solutions. Data synchronization becomes a stumbling block and, as a result of all of these issues, he believes that decentralization is impractical. He went to explain why he does not believe bitcoin is a currency, although he did admit that this is a grey area. He stated that things are categorized by how they behave; e.g. Real-estate sold on an allocation basis is a security. He argued that bitcoin is about buying shares that can then be sold for money and that there are strong arguments being made to regulate Bitcoin as a security, not as a currency.

His stated that he believes in the power of "norms" such as community standards (people tend to imitate what they see most people doing); shared goals and expectations; shared guardrails such as the Ten Commandments; trust/identity (social security numbers); and laws and regulations (everyone accepts the fact that a red light means stop and that a green light means go - social norms are powerful, although Anderson said that social norms are not always good).

I believe that the essence of Anderson's talk can be summed up by the closing of his blog post in the The Scholarly Kitchen that was noted earlier. "Is blockchain relevant to scholarly communication? Possibly, but it's important to realize that it's far from plug-and-play, that legal issues aren't settled, and that depending on the use-case, it may not be the best technical solution if you're trying to balance easeof-implementation, costs, security, compliance, and many other factors. To borrow a phrase used earlier, the substance of an activity matters more than the form it takes.

Or, to quote Douglas Adams, "We are stuck with technology when what we really want is just stuff that works [22]". Needless to say, there were a lot of back-and-forth comments after the presentation! But Anderson's presentation re-enforced Griffey's comment about blockchain being at the peak of the technology hype cycle and served as a great counter-balance to some of the talks that both preceded (and followed) his.

Anderson's slides are available on the NFAIS website. He has given approval to reprint his posting in The Scholarly Kitchen and it appears as an article elsewhere in this issue of Information Services and Use.

The lunch keynote speaker was Petr Novotny, Research Staff Member, IBM Thomas J. Watson Research, who spoke on the impact of blockchain on Scholarly Publishing and the importance of developing a strategy for managing digital assets. He said that blockchain technology will do for trusted transactions what the Internet did for information. As Information Technology moves to the Cloud, transactions must be trusted by all parties involved because the global digital economy depends on trust, not faith.

As noted throughout the conference, blockchain brings together shared ledgers with smart contracts to allow the secure transfer of any asset, from a physical asset such as a shipping container to financial assets such as bonds or a digital asset such as scholarly content - across any business network. Transparency of transactions, as well as the immutability of the shared blockchain ledger, are key features when it comes to proofing the provenance of assets and allowing permissions-based authentication.

Novotny went on to describe some of the IBM blockchain projects that are currently in progress. As of March 10, 2018 there were four hundred projects in partnership with sixty-three organizations (see: https://bitsonline.com/ibm-blockchain-projects/). These include using blockchain to address food safety in partnership with Walmart and nine other sources (The Food Trust Blockchain), the goal of which is 
to improve the companies' ability to identify issues involved with food recalls, such as tracing outbreaks more quickly to limit customer risk.

Walmart's vice president of food safety, Frank Yiannas, referred to the Food Trust blockchain as the "equivalent of FedEx tracking for food [23]". Another includes tracking diamonds across the globe in support of ethical trade [24].

He then went on to describe how IBM is now offering a managed blockchain as a service (BaaS). As one of the pioneers in this area, IBM developed the IBM Blockchain Platform (IBP), a full stack blockchain-as-a-service offering with features that include high performance and enhanced security, and is available in a globally-distributed public cloud, analogous to similar cloud offerings from Amazon and Microsoft. He said that BaaS simplifies and accelerates the adoption of blockchain technologies for many industries, including academic publishing. Currently, a large number of academic publishing processes and many other related activities are managed on various disconnected platforms. BaaS can help to effectively integrate the academic publishing domain. Novotny then went on to describe the technical infrastructure of this new IBM service.

In closing, Novotny said that blockchains provide the opportunity and building blocks to design new solutions, which will address shortcomings of the present technologies as well as significantly transform and extend the existing processes and modes of operation to the benefit of the academic publishing community.

Novotny's slides are available on the NFAIS website and a paper that gives an overview of both blockchain technology and IBM's applications appears elsewhere in this Issue of Information Services and Use. A special treat for conference attendees and readers of this Issue of Information Services and Use, is a complimentary copy of IBM's 2nd edition of Blockchain for Dummies that is freely-accessible at: https://www-01.ibm.com/common/ssi/cgi-bin/ssialias?htmlfid=XIM12354USEN.

The fist speaker of the afternoon was Patrice Lyons, Esq., General Counsel, Corporation for National Research Initiatives (CNRI), who gave a fascinating talk on blocks as digital entities from a historical perspective, especially with regards to the many related CNRI activities. In the mid-1980s, CNRI developed the concept of mobile programs called Knowbots and issued a seminal report describing this technology [25]. Knowbots were originally envisioned and implemented by CNRI as objects in the sense of object-oriented programming. CNRI continued to evolve the original Knowbot technology by developing an alternate approach to the implementation of mobile programs structured as Digital Objects which became the basis for the Digital Object (DO) Architecture. She went on to describe the fundamental properties of this architecture and noted that it is based upon the same important architectural ideas that underpin the Internet more generally, the three most important of which are: Open Architecture with defined protocols and interfaces; independence from the underlying technology; and minimized complexity for users. She added that the early definition of the Internet by the U.S. Federal Networking Council in 1995 anticipated that the basic Internet technology would continue to evolve over time, and she suggested that attendees read "A Brief History of the Internet" to see the text of the resolution [26].

Lyons went on to define a "Digital Object (DO)" as a sequence of bits, or a set of such sequences, having an associated unique persistent identifier that can be resolved to state information about the DO, including its location(s), access controls, time of creation, public keys (if any), and verification information. A DO incorporates a work or other information in which a party has rights or interests, or in which there is value. She noted that any kind of information in digital form may be structured as a digital object. The notion of a digital object may be viewed as a logical extension of the notion of a packet in a network context. Each DO has an associated unique persistent identifier that enables the DO to be accessed in the Internet 
or other computational facilities by resolving its identifier. The DO can be signed and validated using the Public Key Infrastructure (PKI) or other cryptographic methods intrinsic to the overall DO Architecture.

Based largely on the Digital Object Architecture, ITU-Recommendation X.1255: Framework for discovery of identity management information [27] was approved in September 2013 by the International Telecommunication Union. For purposes of X.1255, a digital object is defined as a digital entity and the Recommendation describes a data model and interface protocol. A recent meeting of the ITU Focus Group on Application of Distributed Ledger Technology that was held in Bern, Switzerland in February of this year addressed the CNRI contribution on the definition of "blocks" as "digital entities", and this was noted in the description of "block" in the relevant outcome document [28].

Like Ken Anderson, Lyons also noted that blocks are not new! Historically, a block, also called a data structure, was viewed essentially as a sequence of bits, usually with a defined beginning and end. In the past, it may or may not have been uniquely identified other than, perhaps, by its arrival sequence in time. Blocks were also linked with other blocks. For example, in the programming field, blocks were often linked or chained using pointers, and in the communications field they were usually linked in some time sequence and often involved encryption. Blocks were not usually managed separately from the application that invoked them - but they could be.

She went on to say that a block may be viewed as a digital entity that is configured using specific methods and that there are many issued and pending patents on various ways to accomplish this. Immutable digital entities can be authenticated without reliance on external parties and some examples are transactions, contracts, bills of lading. Mutable objects rely on external mechanisms to validate, and while blocks may generally be immutable, blockchains may not change, but are inherently mutable. New techniques and applications such as those based on digital entities (including various blockchain implementations) are being developed. When evaluating this technology, past technical developments in related areas such as hashing algorithms, bucket sorting, queuing theory, data typing, and, in particular, advanced computer programming techniques are also potentially relevant.

As the Internet confronts increased complexity, mobile program technology, whether structured as blocks or, more generally, digital entities, may soon be needed in the context of implementations of the DO Architecture. While techniques such as layering or end/end interaction will continue to have utility, a more integrated perspective is appropriate when autonomous devices are widely-deployed and are capable of acting on behalf of users or programs to produce desired results. The complexities and sheer volume of information that will be available requires a new paradigm for information management, and the Digital Object Architecture should provide a sound basis for moving forward.

Lyons' closing message was a wise one: It is important to know what has gone before and build on $i t$. Her slides are on the NFAIS website and a more detailed article based upon her presentation appears elsewhere in this issue of Information Services and Use.

The next speaker was Sonia Mundra, President, Chenega Analytic Business Solutions (CABS) who spoke on the use of blockchain technology in the development of strategic initiatives. She said that all professionals should consider the use of blockchain as a new and exciting technology when it comes to program risk management. In fact, in January of this year the National Institute of Standards and Technology (NIST), published a draft paper discussing and defining blockchain and its role in security [29]. (Note: I took a quick look at the document and it is definitely worth a read by anyone who needs a primer on blockchain).

Mundra said that when we want to implement a new technology, we need to first understand the capabilities of that technology and exactly what we hope to accomplish by using it - only then can it be implemented in way that will reduce the most risk to the organization. While she encourages everyone 
to consider the use of blockchain technology, she said to do some homework before moving forward. Such homework should include determining if blockchain encryption is a good fit for the organization's digital assets (using a flowchart), determining if the organization is ready for the change (change management) and the role of training as bookends to the program (first initial training, then development of standard operating procedures and user training once the blockchain has been deployed). These steps are outlined in a report issued by the American Council for Technology - Industry Advisory Council (ACT-IAC) that is freely-available [30].

In closing she emphasized the fact that blockchain technology is expensive and strongly recommended that before jumping into any implementation an organization is comfortable that there will be a return on the investment that they are making. It may not be a financial return - it could be that the technology provides far more security for their data than what is currently in place and that the increase in protection is worth the financial investment.

Mundra's slides are not available on the NFAIS website, but a brief article derived from her presentation appears elsewhere in this issue of Information Services and Use.

The next speaker was Dr. Conrad Barski, CEO, Forward Blockchain, LLC (see: http://www.forwardblockchain.com/), a company that focuses on medical applications for blockchain technology. His focus was primarily on blockchain security and he opened by saying that the digital signing mechanism in Bitcoin and Ethereum is the most secure such mechanism ever created. He went on to describe the process of sending a "message" via this technology using a public blockchain platform such as Ethereum. The Ethereum platform has the capability of creating "smart contracts", that help the user exchange money, property, shares, or anything of value in a transparent, conflict-free way while avoiding the services of a middleman. The contracts define the rules and penalties around an agreement in the same way that a traditional contract does, e.g. rules such as who can access the "message", what they can do when they access it, etc., but the smart contract can also automatically enforce those obligations. Public blockchains are secure, reliable, and have zero infrastructure. One uses a private "key" to confirm that they are a valid, authorized user and once created, the contract is pushed into the Ethereum network.

Barski went on to say that the issue with public blockchains is that people are concerned with privacy, especially with regards to medical information. One easy way to alleviate this concern is to build a firewall around the blockchain, although there are other alternatives such as the use of public chain encryption, blockchain permission systems, factom-like systems, zero-knowledge systems, plasma chains, and payment/state channels. He went on to say that in the next five years he believes that the addition of a second peer-to-peer "layer" will be added to public blockchains as a means of securing privacy and offering even greater system performance. He said some examples of second layer systems are sharding (a form of database partitioning), Casper, and Plasma (for more information on these see https://medium.com/futuresin/ethereum-eth-blockchain-ready-for-second-phase-in-q42018-50189b799a7e).

In closing, Barski gave an example of how a second layer system could be overlaid on a blockchain for publishing. He said that such a system would be less of an "application" and more of a "utility." Trusted Authorities would be authorized to access the platform. The platform would decouple publishing, hosting, payment handling, and content validation, and could be optimized for highly-dynamic datasets (mailing lists, research study data, etc.), with automatic support for subscriptions. Systems for scholarly publication in the near future, if they adopt modern ideas such as blockchain technology, will look very different from today's systems. Data will be stored in many different places and many different forms and will be aggregated in new and sophisticated ways. This future is also a place where server systems will 
play a more limited role than they do today, with emphasis once again being placed on powerful client devices.

Barski' slides are available on the NFAIS website.

The closing speaker of the conference was Dr. Sönke Bartling, Founder, Blockchain for Science (see: https://www.blockchainforscience.com/). The mission of his organization is to open up Science and knowledge creation by means of the blockchain (r)evolution. He opened his talk, like those before him, with an overview of the blockchain technology. He compared it to the Internet, saying that while the Internet is a connection of computers, a blockchain is a connection of time stamps. It doesn't tell you exactly what happened, but it does tell you when something has happened (a sentiment voiced by prior speakers). It offers a new way of organizing online servers that is distributed, decentralized, immutable, and transparent. It provides a new way of looking at user identity and autonomy, as well as a new way of looking at computer security. And it detaches control over hardware from control over content.

He went on to say that when we talk about blockchain being decentralized, it is not really a technical decentralization, but rather the lack of an intransparent central point of failure in the system. The service provider and control over the service are mutually independent, offering the possibility of eliminating artificially-constraining business models. In fact, he says blockchain has created a new economy in the form of cryptocurrency. It provides bullet proof variables (tokens, coins) with provable behavior based upon "smart contracts", at near zero costs, and eliminates the need for a trusted third party (paper contract, lawyer, notary, banks, stock exchanges, etc.) is not required. But it has yet to be proven that such an economy will create novel value propositions and an open economy where only a few become really rich. He added that blockchain could very well be an unnecessary technology, but he believes that this will be true only in cases where a perfectly trusted third party exists.

Bartling then switched gears to talk about blockchain and science. He said that the problems in science today are due to scientists themselves and the current science culture. He described the current science environment as one that is burdened by legacy structures. It is a centralized landscape (data repositories, service providers), marked by unlimited media breaches, with walled gardens/silos around business models and content. Research involves a significant amount of administrative effort and competition (especially for grants), resulting in a lot of overhead. In addition, only parts of the research process are really open and most control is in the hands of third parties. He added that the culture of science is deeply intertwined with society and individual's lives. Science is important and fun. The current problem is the traditional culture of science and scientists can be their own worst enemies.

But the culture is changing. Today we have Open Science, Science 2.0, Open Access, and other initiatives. This time even science funding is being affected by both technology and policy. We heard earlier from Jason Barkerloo about how Knowbella Tech is connecting scientific researchers with funding sources via blockchain technology. And, although previously forbidden, as of March 2017 NIH guidelines state that interim research products, including preprints and the pre-registration of protocols, can now be cited anywhere research products are cited, although DOI's are required so that there is a sense of permanence. The full new grant guidelines on this issue can be accessed at: https:/grants.nih.gov/grants/guide/noticefiles/NOT-OD-17-050.html. The change in funding is important to be aware of he said, because where the money goes, the honey goes!

Bartling went on to describe the Research Circle, saying that the following steps can all be connected to form a circle: the generation of an idea/concept, funding, the experiment, acquisition, processing, analysis, and publication. He added that all of these steps could be "blockchained" - not unlike what ARTiFACTS (discussed earlier) is trying to accomplish. Research data in a blockchain is time-stamped and immutable - of critical importance when attempting to obtain a patent. He reinforced that fact that 
processing in a blockchain can be accomplished through smart contracts that are unchangeable and that provide a provable processing trail. Research data could be stored in a decentralized cloud with $100 \%$ cryptography. And there would be the opportunity for dynamic publications with micro-contributions. Even peer-review can be accomplished on the blockchain with the added benefit of offering incentives to those who perform the peer-review, and he noted the same peer review project that I mentioned earlier (see: https://www.blockchainpeerreview.org/).

Bartling returned to the topic of funding and pointed out that ICOs (initial coin offerings) [31] have now been introduced into the research ecosystem. One such offering is ARNA Panacea, a biotech decentralized solution, powered by blockchain and ARNA tokens, that offers a platform for the processing of clinical trials and support to researchers during the research and development phase (see: https://token.arnagenomics.com/).

He said that we have all of the pieces for a blockchain research infrastructure: we have trusted third parties; no mining is necessary; we already have the best mining financial system in Bitcoin; and we can combine different blockchains for ongoing development. He asked (rhetorically) how much technical decentralization does science really need. Centralized, non- blockchain services will coexist, for worthless and or minor transactions.

Bartling closed by saying that now is the time to aggressively move forward with the new culture of science, and he mentioned the First International Conference on Blockchain for Science and Knowledge Creation that is being held 5-6 November 2018 in Berlin, Germany see: https://www.blockchainforsciencecon.com/.

\section{Conclusion}

I thoroughly enjoyed the conference and learned a lot. I consider myself a publisher and a product developer, not a technologist, so some of the presentations forced me to find additional reading so I could put speakers' comments in context [32]. On the other hand, I admit to being a blockchain enthusiast have been since last year. When conversing with other publishers who attended this conference, I found that most were like me. They came to find out how others perceived blockchain technology and if/how they were utilizing it (most were not - they were looking at how it could be best applied within their own organization and attempting to learn from others who might already be doing so). But again, like me, they were all mesmerized by its potential.

As you probably noted, Eugene Garfield was mentioned several times by speakers. I worked with and for him for almost thirty years at what was then the Institute for Scientific Information (ISI), and I guarantee if he were here today he would be seriously thinking about how ISI could best utilize the technology. He could "smell" good technologies when they were in their infancy (admittedly, most of us at ISI, while good at "smelling" great technologies, were not always good at judging when the market itself would be ready for adoption - a critical time-to-market issue). And that is my takeaway from this meeting.

For while I was seriously impressed by how many blockchain-based initiatives are already underway and how much consensus there was among the speakers on what the technology can potentially bring to the publishing arena, I am not sure if others involved in the publishing chain - from lab bench researcher to publisher to information consumer - are ready for adoption (and if they are even aware of the technology). And it is their ultimate adoption that is essential to the success of the initiatives. I tend to agree with Jason Griffey's comment that blockchain technology is "at the peak of inflated expectations" since most 
of the initiatives discussed are very new. But I do firmly believe that it is one of the technologies that will eventually emerge to the plateau of productivity - e.g. success [33]!

From tracking the generation of a research concept, through funding, experimentation and collaboration, analysis of results, manuscript generation, peer review, and publication itself - even to the creation of a blockchain-based citation index, the technology appears to be a perfect match with scholarly publishing. I wish ARTiFACTS the best of luck - I love their concept! Indeed, the technology may even eventually create the publisher - content creator ecosystem that Mads Holmen recommended. But as enthusiastic as I am, I believe that everyone involved needs to keep in mind some of the pearls of wisdom that were sprinkled throughout the presentations:

- Blockchain is not a solution in search of a problem; while worth exploring, implementation is not for everyone. Do your homework first!

- Blockchain is a potential disruptor for the information economy; therefore publishers - the middlemen between authors and information consumers - need to take this technology very seriously and use it to their advantage.

- Blockchain does offer an opportunity to create the ultimate citation index (it will allow the inclusion of the long-tail of published articles). Citation index publishers should think about how to leverage the technology to their advantage.

- Blockchain has been built on prior technologies, many of which are still essential. It is important to know what has gone before and to continue to build upon the strongest technologies.

Perhaps the most important pearl of wisdom is that of which Jason Griffey reminded us - Amara's Law:

"We tend to overestimate the effect of a technology in the short run and underestimate the effect in the long run [34]".

My advice - do not under estimate the potential of blockchain technology in the long run!!!! I love horse racing - and if it were a horse, I would bet on it!

Note: If permission was given to post them, speaker slides used during the NFAIS Conference on Blockchain and Scholarly Publishing are embedded within the conference program at: https://nfais.memberclicks.net/2018-blockchain-program. The term "slides", if they are available, is highlighted in blue.

Also, plan on attending the 2019 NFAIS Annual Conference that will take place in Alexandria, VA, from February 13-15, 2019. Watch for details on the NFAIS website at: http://www.nfais.org/.

\section{About the Author}

Bonnie Lawlor served from 2002-2013 as the Executive Director of the National Federation of Advanced Information Services (NFAIS), an international membership organization comprised of the world's leading content and information technology providers. She is currently an NFAIS Honorary Fellow. Prior to NFAIS, Bonnie was Senior Vice President and General Manager of ProQuest's Library Division where she was responsible for the development and worldwide sales and marketing of their products to academic, public, and government libraries. Before ProQuest, Bonnie was Executive Vice President, Database Publishing at the Institute for Scientific Information (ISI - now Clarivate Analytics) where she was responsible for product development, production, publisher relations, editorial content, and worldwide sales and marketing of all of ISI's products and services. She is a Fellow and active 
member of the American Chemical Society and a member of the Bureau of the International Union of Pure and Applied Chemistry for which she chairs their Publications and Cheminformatics Data Standards Committee. She is also on the Board of the Philosopher's Information Center, the producer of the Philosopher's Index, and she serves as a member of the Editorial Advisory Board for Information Services and Use. She has served as a Board and Executive Committee Member of the former Information Industry Association (IIA), as a Board Member of the American Society for Information Science \& Technology (ASIS\&T), and as a Board member of LYRASIS, one of the major library consortia in the Unites States.

Ms. Lawlor earned a B.S. in Chemistry from Chestnut Hill College (Philadelphia), an M.S. in chemistry from St. Joseph's University (Philadelphia), and an MBA from the Wharton School, (University of Pennsylvania).Contact: E-mail: chescot@aol.com.

\begin{abstract}
About NFAIS
Founded in 1958, the National Federation of Advanced Information Services (NFAIS ${ }^{\mathrm{TM}}$ ) is a global, non-profit, volunteer-powered membership organization that serves the information community; i.e., all those who create, aggregate, organize, and otherwise provide ease-of-access to and effective navigation and use of authoritative, credible information.

Member organizations represent a cross-section of content and technology providers, including database creators, publishers, libraries, host systems, information technology developers, content management providers, and other related groups. They embody a true partnership of commercial, nonprofit, and government organizations that embraces a common mission - to build the world's knowledgebase through enabling research and managing the flow of scholarly communication.

NFAIS exists to promote the success of its members and for more than sixty years has provided a forum in which to address common interests through education and advocacy.
\end{abstract}

\title{
References
}

[1] Bonnie Lawlor, An overview of the NFAIS 2017 Annual Conference: The big pivot: Re-engineering scholarly communication, Information Services and Use 37(3) (2017), 283-306, https://content.iospress.com/journals/information-servicesand-use/37/3?start=10n (last accessed July 28, 2018).

[2] N.J. Casey and P. Vigna, In blockchain we trust, MIT Technology Review 121(3) (2018), 10-16.

[3] M. Baker, 1,500 scientists lift the lid on reproducibility, Nature (2016), https://www.nature.com/news/1-500-scientistslift-the-lid-on-reproducibility-1.19970 (last accessed 28 July 2018).

[4] D. Yaga, P. Mell, N. Roby and K. Scarfone, NISTIR 8202 Blockchain Technology Overview National Institute of Standards and Technology, U.S. Department of Commerce, 2018.

[5] J. Van Rossum, Blockchain for Research: Perspectives on a new Paradigm for Scholarly Communication, Digital Science Report, November 2017, https://figshare.com/articles/_/5607778, (last accessed 3 July 2018).

[6] S. Nakamoto, Bitcoin: A Peer-to-Peer Electronic Cash System, May 24, 2009 (see: https://web.archive.org/web/20140320135003/https://bitcoin.org/bitcoin.pdf). Access on 8 September 2018.

[7] B. Lawlor, An overview of the NFAIS 2017 Annual Conference: The big pivot: re-engineering scholarly communication, Information Services and Use 37(3) (2017), 300, For more information on this journal refer to the following article: https://content.iospress.com/journals/information-services-and-use/37/3?start=10 (last accessed 8 September 2018).

[8] Sci-Hub, see: https://en.wikipedia.org/wiki/Sci-Hub, accessed 17 September 2018.

[9] ResearchGate, see: https://en.wikipedia.org/wiki/ResearchGate, accessed 17 September 2018.

[10] See: https://snakemake.readthedocs.io/en/stable/, accessed 8 September 2018.

[11] Space Elevator, https://en.wikipedia.org/wiki/Space_elevator, accessed 8 September 2018. 
[12] Technology Hype Cycle, see: https://en.wikipedia.org/wiki/Hype_cycle, accessed 16 September 2018.

[13] Roy Amara, https://en.wikipedia.org/wiki/Roy_Amara, accessed 9 September 2018.

[14] RG Score, https://explore.researchgate.net/display/support/RG+Score, accessed 9 September 2018.

[15] E. Garfield, The science citation index: A new dimension in indexing, Essays of an Information Scientist 7: (1984), 525535, https://pdfs.semanticscholar.org/992c/22c3ddcc142c63be1edbd7584bf2d983546a.pdf, accessed 10 September 2018.

[16] S.C. Bradford, Documentation. Public Affairs Press, Washington, DC, 1950.

[17] E. Garfield, The mystery of transposed journal lists - wherein Bradford's law of scattering is generalized according to Garfield's law of concentration, Essays of an Information Scientist 1: (1971), 222-223, Current Comments 17.

[18] R. K. Merton, FORWARD, Essays of an Information Scientist 4: (1981), 218-221, http://www.garfield.library.upenn.edu/essays/v4p216y1979-80.pdf, accessed 10 September 2018.

[19] K. Anderson, Can blockchain withstand skepticism? An inquiry, The Scholarly Kitchen (2018), https://scholarlykitchen.sspnet.org/2018/04/05/can-blockchain-withstand-skepticism-an-inquiry/, accessed September 10, 2018.

[20] P. Ford, Bitcoin is ridiculous; blockchain is dangerous, Bloomberg Businessweek, March, 9, 2018, https://www.bloomberg.com/news/features/2018-03-09/bitcoin-is-ridiculous-blockchain-is-dangerous-paul-ford, accessed 10 September 2018.

[21] B. Adida, Blockchain and Voting, Benlog, December 28, 2017, https://benlog.com/2017/12/28/blockchain-and-voting/, accessed 19 September 2018.

[22] D. Adams, The Salmon of Doubt: Hitchhiking the Galaxy one Last Time. Ballantine Books, New York, 2003.

[23] M.J. Zuckerman, Walmart, IBM Blockchain Initiative Aims to Track Global Food Supply Chain, https://cointelegraph.com/news/walmart-ibm-blockchain-initiative-aims-to-track-global-food-supply-chain, accessed 14 September 2018

[24] J.J. Roberts, IBM Blockchain is Tracking Diamond Tings Across the Globe, Fortune, April 26, 2018, http://fortune.com/2018/04/26/ibm-blockchain-diamonds-helzberg/, accessed September 14, 2018.

[25] R.E. Kahn and V.G. Cerf, An Open Architecture for a Digital Library System and a Plan for Its Development, The Digital Library Project Volume I: The World of Knowbots, (DRAFT) March 1988, http://hdl.handle.net/4263537/2091. Accessed September 11, 2018.

[26] For Federal Networking Council's Resolution defining the Internet (1995), see "A Brief History of the Internet," ACM SIGCOMM Computer Communications Review, 39, 5, October 2009, at page 30, https://www.cs.ucsb.edu/ almeroth/classes/F10.176A/papers/internet-history-09.pdf, accessed 11 September 2018.

[27] ITU Recommendation X. 1255, "Framework for discovery of identity management information" is based largely on CNRI's Digital Object Architecture and Robert E. Kahn, CNRI's President, served as Editor, https://www.itu.int/rec/TREC-X.1255-201309-I, accessed 11 September 2018.

[28] Contribution of Corporation for National Research Initiatives, DLT-I-048, Definition of "block" as a "Digital Entity," ITU Focus Group on Application of Distributed Ledger Technology, Bern, Switzerland (February 2018), http://www.cnri.reston.va.us/documents/DLT-I-048_CNRI_Contribution_FG_DLT.pdf, accessed 11 September 2018.

[29] D. Yaga, P. Mell, N. Roby and K. Scarfone, NISTIR 8202 Blockchain Technology Overview National Institute of Standards and Technology, U.S. Department of Commerce, 2018, https://csrc.nist.gov/publications/detail/nistir/8202/draft, accessed 12 September 2018.

[30] Enabling Blockchain Innovation in the U.S. Federal Government: A Blockchain Primer, American Council for Technology - Industry Advisory Council (ACT-IAC), October 17, 2017, https://www.actiac.org/act-iac-white-paper-enablingblockchain-innovation-us-federal-government, accessed September 12, 2018.

[31] "Initial Coin Offering" - a type of funding using cryptocurrencies, https://en.wikipedia.org/wiki/Initial_coin_offering, accessed 14 September 2018.

[32] Consider reading The Truth machine: the Blockchain and the Future of Everythingby Michael J. Casey and Paul Vigna, St. Martin's Press, 175 Fifth Avenue, New York, NY, USA, February 2018 and Blockchain Basics: A Non-Technical Introduction in 25 Steps by Daniel Drescher, Apress, 2017.

[33] Gartner Hype Cycle. See: https://whatis.techtarget.com/definition/Gartner-hype-cycle, accessed 17 September 2018.

[34] Roy Amara, https://en.wikipedia.org/wiki/Roy_Amara, accessed 9 September 2018. 